\title{
A proposed nosology of inborn errors of metabolism
}

\author{
Carlos R. Ferreira, MD ${ }^{1,2}$, Clara D. M. van Karnebeek, MD, $\mathrm{PhD}^{3,4}$, Jerry Vockley, MD, $\mathrm{PhD}^{5}$ and \\ Nenad Blau, PhD ${ }^{6}$
}

Purpose: We propose a nosology for inborn errors of metabolism that builds on their recent redefinition.

Methods: We established a strict definition of criteria to develop a self-consistent schema for inclusion of a disorder into the nosology.

Results: We identified 1015 well-characterized inborn errors of metabolism described in the literature. In addition, there are 111 less well-characterized conditions that may be inborn errors but do not meet strict criteria for inclusion in the current nosology.

Conclusion: We provide a master list of all currently recognized inborn errors of metabolism grouped according to their pathophysiological basis, with the hope of setting a standard against which new errors should be defined, as well as to promote awareness and foster collaboration in the area. With the rapid advances in the field of genetics in recent years, it is likely that this nosology will need to be updated in the near future, a process that will benefit from broader input and collaboration of experts in the field to improve future versions of the proposed classification.

Genetics in Medicine (2019) 21:102-106; https://doi.org/10.1038/ s41436-018-0022-8

Key Words: Nosology; Inborn errors of metabolism; Inherited metabolic disorders; Classification.

\section{INTRODUCTION}

The first inborn error of metabolism (IEM) was described in 1902 by Sir Archibald Garrod. ${ }^{1}$ Since then, many more have been described, variously estimated as $>600$ (ref. ${ }^{2}$ ) or $>700$ $\left(\right.$ ref. $\left.^{3}\right)$. However, the exact number of IEMs first begs the question: what is an IEM? This subject has recently been addressed elsewhere. ${ }^{4}$ In the era of clinical genomics, IEMs can no longer be considered as only monogenic conditions involved in the synthesis or catabolism of molecules within a specific pathway, leading to measurable abnormalities in classic biochemical laboratory methods. Rather, it has been suggested that an IEM should instead be defined to include any condition in which the impairment of a biochemical pathway is intrinsic to the pathophysiology of the disease.

A nosology is a classification of diseases. Nosologies for other groups of disorders have grappled with issues of definition. For example, a nosology of skeletal dysplasias was first introduced almost 50 years ago, ${ }^{5}$ it has been regularly updated ever since, and most recently is in its ninth iteration. ${ }^{6}$ Similarly, the first classification of primary immunodeficiencies was also proposed in the $1970 \mathrm{~s}^{7}$ after the World Health Organization sponsored a Primary Immunodeficiency Expert Committee to classify human primary immune defects. Once established, a nosology provides a framework for diagnosing, studying, and treating a group of disorders. A formal attempt to systematically establish a nosology of IEMs, as well as to continuously curate its content to maintain its relevance, has never been proposed. The purpose of the present article is to develop such a nosology, and to apply it to the recently established Inborn Errors of Metabolism Knowledgebase (IEMbase). ${ }^{8}$

\section{MATERIALS AND METHODS}

We first established specific criteria to classify a disorder as an IEM. These criteria are summarized in Table $\mathbf{1}$, and explained in detail here.

In the current nosology, we ascribe to the recent redefinition of an IEM as any condition that leads to a disruption of a metabolic pathway, irrespective of whether it is associated with abnormalities in biochemical laboratory tests. We not only include primary enzyme or transporter deficiencies or superactivities, but also chaperone deficiencies-such as DNAJC12 deficiency presenting with hyperphenylalaninemia-or deficiencies in transcription factors that can induce metabolic abnormalities, such as HCF1 deficiency with methylmalonic aciduria, GATA1 deficiency with excess uroporphyrin I excretion, or NRF2 superactivity with hypohomocysteinemia.

We define single entries for individual enzyme deficiencies regardless of severity. For example, we do not include three

\footnotetext{
${ }^{1}$ National Human Genome Research Institute, National Institutes of Health, Bethesda, Maryland, USA; ${ }^{2}$ Division of Genetics and Metabolism, Children's National Health System, Washington, DC, USA; ${ }^{3}$ Departments of Pediatrics and Clinical Genetics, Academic Medical Centre, Amsterdam, The Netherlands; ${ }^{4}$ Department of Pediatrics, Centre for Molecular Medicine and Therapeutics, University of British Columbia, Vancouver, BC, Canada; ${ }^{5}$ Department of Pediatrics, University of Pittsburgh School of Medicine, Department of Human Genetics, Graduate School of Public Health, Pittsburgh, Pennsylvania, USA; ${ }^{6}$ Dietmar-Hopp Metabolic Center, University Children's Hospital, Heidelberg, Germany. Correspondence: Carlos R. Ferreira (carlos.ferreira@nih.gov)
} 
Table 1 Criteria used for inclusion of an inborn error of metabolism in the current nosology

The disruption of a metabolic pathway is considered necessary and sufficient for inclusion

Regardless of laboratory abnormalities in standard biochemical tests

Regardless of association with clinical manifestations of disease (unless the defect is universal to all humans)

Severity alone is not considered sufficient for separation into different entries when a single gene product is involved

A different pathomechanism is considered necessary for separation into different entries when a single gene product is involved, regardless of the mode of inheritance

The involvement of different gene products is considered sufficient for separation into different entries, even if the phenotype is similar

The error must have been reported in more than a single family, and the involvement of the gene product must have been well characterized on an enzymatic or molecular level

separate entries for Gaucher disease types 1, 2, and 3, as this classification is largely dependent on severity of the enzyme deficiency, and not on a different pathophysiology. Similarly, there is only one entry for lysosomal acid lipase deficiency, and not two separate entries for Wolman disease and cholesteryl ester storage disease. However, we provide the alternative nomenclature for these disorders in a separate column. On the other hand, single enzyme deficiencies are included as two separate entries when they lead to clearly delineated phenotypes, not strictly associated with severity. For example, GM1 gangliosidosis and Morquio syndrome type B are both caused by $\beta$-galactosidase deficiency, but the phenotype is clearly distinct. Similarly, a deficiency of acid ceramidase can lead to Farber disease (of variable severity), but also to a different phenotype of spinal muscular atrophy with progressive myoclonic epilepsy, with no known correlation between the degree of enzyme activity and the phenotypic presentation. In the case of ATP7A-related distal motor neuropathy, the clinical and metabolic findings are completely unrelated to those of Menkes disease or occipital horn syndrome, and in fact the neuropathy phenotype is caused by distinct variants leading to a distinct pathomechanism. Such situations generate separate entries for defects in the same protein.

Conditions that can have both autosomal recessive and autosomal dominant inheritance are included under the same entry as long as they share a similar mechanism of disease, as is the case with MCT1 deficiency, SCOT deficiency, 5-oxoprolinase deficiency, ISCU deficiency, or hereditary hyperekplexia type 1 . However, in some disorders, the different pattern of inheritance is associated with a completely different phenotype; for example, biallelic variants in genes encoding the subunits of succinate dehydrogenase lead to a leukodystrophy, while heterozygous variants in the same genes lead to familial paragangliomas, the latter likely via inhibition of HIF prolyl hydroxylases. ${ }^{9}$ For some disorders, the line is somewhat blurry. For example, autosomal dominant GTP cyclohydrolase deficiency manifests as doparesponsive dystonia without hyperphenylalaninemia, while the autosomal recessive form manifests with earlier onset of severe neurologic dysfunction and hyperphenylalaninemia due to a more severe and systemic tetrahydrobiopterin deficiency. Thus, the current separation of this enzymatic defect into two separate entries is maintained, based on different clinical and biochemical findings, and different management. However, intermediate phenotypes do exist, as patients have been described with biallelic variants in $G C H 1$ but only exhibiting dopa-responsive extrapyramidal symptoms without hyperphenylalaninemia, ${ }^{10,11}$ while some heterozygous carriers exhibit hyperphenylalaninemia when given a phenylalanine load but otherwise are asymptomatic. ${ }^{12}$ Similarly, autosomal dominant hypermethioninemia caused by dominant negative variants in MAT1A is given a separate entry from the autosomal recessive MAT I/III deficiency caused by loss-of-function variants. In the case of $\Delta-1$ pyrroline-5-carboxylate synthase deficiency, a predominant spastic paraplegia phenotype can be seen both with autosomal dominant or recessive inheritance, while a phenotype that includes neurologic involvement and cutis laxa can also be inherited in either an autosomal dominant or recessive fashion. The dominant inheritance of either the cutis laxa phenotype or the spastic paraplegia phenotype is likely due to dominant negative variants. ${ }^{13,14}$ In such cases, we include two entries for the same enzyme deficiency based on the clinical phenotype (as opposed to four separate entries, based on both phenotype and inheritance pattern). Classifying X-linked disorders as dominant or recessive can be problematic given the fact that females can have manifestations from X-linked conditions classically defined as recessive. ${ }^{15,16}$ Nevertheless, we elected to include two separate entries for X-linked disorders that present with different phenotypes in heterozygote females as compared with hemizygote males. Examples of this include the $\mathrm{X}$-linked recessive $\mathrm{CK}$ syndrome or $\mathrm{X}$-linked dominant CHILD syndrome, both caused by variants in NSDHL, or the $\mathrm{X}$-linked recessive MEND syndrome versus $\mathrm{X}$-linked dominant chondrodysplasia punctata, both caused by variants in EBP.

Different variants in some proteins can lead to either deficiency or superactivity, with drastically different phenotypes. Examples include the monocarboxylate transporter 1, $\delta$-aminolevulinic acid synthase, phosphoribosylpyrophosphate synthetase, and glucokinase. Additionally, different variants in the same protein can lead to disparate mechanisms of disease. For example loss-of-function variants in the FTL 
gene lead to hereditary L-ferritin deficiency, while gain-offunction variants in exon 4 that alter the reading frame disrupt protein folding and stability and lead to neuroferritinopathy. Additionally, variants in the 5 ' noncoding ironresponsive element (IRE) of the gene lead to hyperferritinemia-cataract syndrome. Such cases are included as separate entries based on the discrepant pathophysiology.

Another criterion used in the current nosology was to include entries based on the particular gene product involved, and not solely on the clinical phenotype. For example, we included separate entries for glycine encephalopathy caused by the deficiency of glycine decarboxylase or aminomethyltransferase. Similarly, defects in many different mitochondrial transfer RNAs can lead to the MELAS phenotype, while defects in multiple nuclear-encoded mitochondrial proteins as well as mitochondrial DNA genes can lead to Leigh syndrome. We include separate entries for involvement of each such gene product.

Numerous biochemical phenotypes that do not cause clinical disease have been described. Pentosuria, one of the first IEMs described by Archibald Garrod during his Croonian lectures, is asymptomatic. ${ }^{17}$ Other IEMs classically considered "nondiseases" include histidinemia, hydroxyprolinemia, or cystathioninuria. In contrast, some biochemical phenotypes originally thought to be clinically significant have been called into question (short-chain acyl-CoA dehydrogenase and 3-methylcrotonyl-CoA carboxylase deficiencies). Finally, some IEMs originally considered not to be clinically relevant have subsequently been associated with a disease phenotype, such as pterin-4- $\alpha$-carbinolamine dehydratase deficiency. ${ }^{18,19}$ All of these conditions have been included in the current nosology, as the term inborn error of metabolism does not by necessity denote a disease. We have not included variants in metabolism that are ubiquitous in humans as compared with other species, for example, lack of Lgulonolactone oxidase or urate oxidase activities.

Whenever possible, entries are named according to the protein involved, unless it is impractical. For example, the name "iminoglycinuria" is well established, and to insist instead on the protein-based name "proton amino acid transporter deficiency with or without imino acid transporter deficiency" is not likely to be generally accepted. Similarly, the name Hartnup disorder is well established, and preferable to " $\mathrm{B}{ }^{0}$ neutral amino acid transporter 1 deficiency."

It should be noted that many entities could be assigned to more than one group based on function and phenotype, a situation that is acknowledged when pertinent. For example, succinate dehydrogenase functions both in the Krebs cycle and as part of the mitochondrial respiratory chain. In such cases, the specific phenotype is included in the group most directly related to the disease mechanism. For example, fructose-1,6-bisphosphatase deficiency is included in the group of disorders of gluconeogenesis, while it is only crossreferenced in the group of disorders of fructose metabolism.

We have not included conditions that remain unpublished, are not verified, or unlikely to have a genetic etiology. These conditions are considered to be poorly characterized either because their molecular basis has not been elucidated, because only a few patients have been described in decades-old reports, or because only individual families have been reported. However, it is possible that these proposed IEMs will be validated in the future.

Finally, certain contiguous gene deletions that include genes encoding for enzymes often have a phenotype distinct from the isolated (single gene) defect. Some examples include the combined deletion of $M A O A$ and $M A O B,{ }^{20-22}$ contiguous $\mathrm{ABCD} 1 / \mathrm{DXS1375E}$ deletion syndrome (also known as CADDS) ${ }^{23}$ hypotonia-cystinuria syndrome, ${ }^{24,25}$ or complex glycerol kinase deficiency. They are not included in the nosology.

\section{RESULTS}

The complete list of IEMs according to our criteria is detailed in Table S1. We define 1015 IEMs in 130 groups. An additional 111 IEMs fall into the final category with lack of substantiation (Table S2). Obviously, the total number of IEMs will vary depending on the inclusion criteria. The most prominent category was that of mitochondrial disorders (groups $54-81$ ) with 232 IEMs (22.9\% of the total). Other important categories included errors of metabolism of nitrogen-containing compounds (groups 1-24) with 188 IEMs (18.5\%), disorders of glycosylation (groups 115-130) with 123 IEMs (12.1\%), disorders of vitamin metabolism (groups 25-38) with 79 IEMs (7.8\%), storage disorders (groups 100-108) with 67 IEMs (6.6\%), disorders of sterol and steroid metabolism (groups 95-97) with 46 IEMs (4.5\%), peroxisomal disorders (groups 109-113) with 27 IEMs (2.7\%), and disorders of metal metabolism (groups 40-43) with 25 IEMs (2.5\%).

\section{DISCUSSION}

Different standard textbooks use different classifications of IEMs, ${ }^{26-28}$ and 610 IEMs are currently tabulated in the IEMbase. ${ }^{8}$ It should be noted that our current nosology was not created with the intention of representing a new coding system. However, the two most commonly used coding systems, the International Classification of Diseases (ICD) and the Systematized Nomenclature of Medicine-Clinical Terminology (SNOMED-CT) are known to have wide gaps in their inclusion of metabolic disorders, which could be filled by the establishment of a formal nosology. In fact, a proposed new coding system created by Dutch clinical specialists found that there were no matches for $76 \%$ of metabolic disorders in ICD-10, and 54\% of metabolic disorders in SNOMED-CT. ${ }^{29}$ OMIM, although comprehensive and authoritative as an online resource for genetic disorders-including inborn errors of metabolism-also does not function as a formal nosology, but rather as a catalog. ${ }^{30}$ On the other hand, there has been a prior attempt to categorize a limited number of metabolic disorders according to their clinical presentation, in order to facilitate diagnosis. ${ }^{31}$ These aforementioned systems did not formally attempt to classify metabolic disorders according to 
their pathophysiological and molecular basis, phenotype, and inheritance.

A special mention should be given to the hierarchical classification established by the Society for the Study of Inborn Errors of Metabolism (SSIEM), accessible via their website (http://www.ssiem.org/resources/IEC.asp) and last updated in 2012. Subsequently, this classification appeared in print in 2014 (ref. ${ }^{32}$ ), and it includes 487 IEMs in 86 disease groups. This classification also grouped IEMs according to their specific biochemical pathways and pathophysiology, and went on to become the basis for sections 5C30 to 5C44 of ICD-11, scheduled to appear in 2018. Unfortunately, this classification has not been updated in years and it includes about half the number of IEMs included in our nosology. In fact, a review of IEMs in the beta draft version of ICD-11 (https://icd.who.int/dev11/1-m/en) reveals a great number of missing IEMs. Our nosology does not only differ from this prior classification in the fact that it's up to date, but also in a number of other features. In particular, we tried to categorize IEMs not only based on the involved pathway and pathomechanism, but also based on the disease phenotype, and on the specific protein or gene product that is deficient, superactive, or dysregulated, all according to carefully delineated inclusion criteria. Another difference is that we decided not to include 111 less well-characterized IEMs, until further confirmation of their validity as unique errors is established based either on enzymatic or molecular grounds for some IEMs, or reports of a second individual or family for others. It is quite likely that many of these less wellcharacterized IEMs will eventually be confirmed, and it is possible that our decision to exclude them from the established nosology could help raise awareness of the fact that, for many of these conditions, further reports are needed for confirmation of the phenotype.

The benefits of having a nosology for IEMs go beyond the purely academic. First, it provides the medical community with an overview of currently recognized IEMs grouped according to their molecular etiology, with the intention that this will set the standard against which potential new IEMs should be defined. Second, it should also assist in identifying patients with ultrarare disorders by providing practitioners a complete list of potential diagnoses. For example, in a patient with hypoglycemia in whom more common causes have been excluded, a quick glance at the group of disorders of insulin secretion can help to notice an ultrarare disorder such as AKT2 superactivity (hypoinsulinemic hypoglycemia with hemihypertrophy). Although not necessarily designed with the intention of being a diagnostic tool, the nosology can act as a quick reminder of the many differential diagnoses for a given IEM, pointing in the appropriate direction for further consultation of appropriate resources, and sets the stage for the development of computer-based, machine learning diagnostic algorithms. Finally, the grouping of conditions can assist in selection of specific genes for sequencing panels or targeted analysis of genome or exome data. Ultimately, we hope that the nosology will promote awareness and foster collaboration among individuals working in the area of metabolism. One of the first applications of the current nosology will be to restructure the IEMbase (www.iembase. org), a knowledgebase of IEMs.

Of course a nosology is only as good as the uniform application of its inclusion criteria, and we believe that the proposed scheme is self-consistent, rather than contradictory. However, any attempt of this type will by necessity be subject to revision as a result of the rapid pace of progress in the field of genetics. Thus, as new disorders are discovered, and our understanding of the pathophysiology of existing disorders continues to improve, frequent updates to the current nosology will be critical to maintain its usefulness. We view the current version as a stepping-stone for improved future iterations, and will facilitate its continuous digital update via the IEMbase.

\section{ELECTRONIC SUPPLEMENTARY MATERIAL}

The online version of this article (https://doi.org/10.1038/s41436018-0022-8) contains supplementary material, which is available to authorized users.

\section{DISCLOSURE}

The authors declare no conflicts of interest.

\section{REFERENCES}

1. Garrod AE. The incidence of alkaptonuria: a study in chemical individuality. Lancet. 1902;160:1616-20.

2. Alfadhel M, Benmeakel M, Hossain MA, et al. Thirteen year retrospective review of the spectrum of inborn errors of metabolism presenting in a tertiary center in Saudi Arabia. Orphanet J Rare Dis. 2016;11:126.

3. Illsinger S, Das AM. Impact of selected inborn errors of metabolism on prenatal and neonatal development. IUBMB Life. 2010;62:403-13.

4. Morava E, Rahman S, Peters V, Baumgartner MR, Patterson M, Zschocke J. Quo vadis: the re-definition of "inborn metabolic diseases." J Inherit Metab Dis. 2015;38:1003-6.

5. International nomenclature of constitutional diseases of bones. Ann Radiol (Paris). 1970;13:455-64.

6. Bonafe L, Cormier-Daire V, Hall C, et al. Nosology and classification of genetic skeletal disorders: 2015 revision. Am J Med Genet A. 2015;167A:2869-92.

7. Cooper MD, Faulk WP, Fudenberg HH, et al. Classification of primary immunodeficiencies. N Engl J Med. 1973;288:966-7.

8. Lee JJY, Wasserman WW, Hoffmann GF, van Karnebeek CDM, Blau N. Knowledge base and mini-expert platform for the diagnosis of inborn errors of metabolism. Genet Med 2017;20:151-58.

9. Selak MA, Armour SM, MacKenzie ED, et al. Succinate links TCA cycle dysfunction to oncogenesis by inhibiting HIF-alpha prolyl hydroxylase. Cancer Cell. 2005;7:77-85.

10. Hwu WL, Wang PJ, Hsiao KJ, Wang TR, Chiou YW, Lee YM. Doparesponsive dystonia induced by a recessive GTP cyclohydrolase I mutation. Hum Genet. 1999;105:226-30.

11. Nardocci N, Zorzi G, Blau N, et al. Neonatal dopa-responsive extrapyramidal syndrome in twins with recessive GTPCH deficiency. Neurology. 2003;60:335-7.

12. Hyland K, Nygaard TG, Trugman JM, Swoboda KJ, Arnold LA, Sparagana SP. Oral phenylalanine loading profiles in symptomatic and asymptomatic gene carriers with dopa-responsive dystonia due to dominantly inherited GTP cyclohydrolase deficiency. J Inherit Metab Dis. 1999;22:213-5.

13. Fischer-Zirnsak B, Escande-Beillard N, Ganesh J, et al. Recurrent de novo mutations affecting residue Arg138 of pyrroline-5-carboxylate synthase cause a progeroid form of autosomal-dominant cutis laxa. Am J Hum Genet. 2015;97:483-92.

14. Panza E, Escamilla-Honrubia JM, Marco-Marín C, et al. ALDH18A1 gene mutations cause dominant spastic paraplegia SPG9: loss of function 
effect and plausibility of a dominant negative mechanism. Brain I Neurol. 2016;139(pt 1):e3.

15. Dobyns WB, Filauro A, Tomson BN, et al. Inheritance of most X-linked traits is not dominant or recessive, just X-linked. Am J Med Genet A. 2004;129A:136-43.

16. Dobyns WB. The pattern of inheritance of $X$-linked traits is not dominant or recessive, just X-linked. Acta Paediatr Suppl. 2006;95:11-15.

17. Garrod AE. The Croonian lectures on inborn errors of metabolism. Lancet. 1908;172:1-7.

18. Ferrè $S$, de Baaij JHF, Ferreira $P$, et al. Mutations in PCBD1 cause hypomagnesemia and renal magnesium wasting. I Am Soc Nephrol. 2014;25:574-86.

19. Simaite $\mathrm{D}$, Kofent J, Gong $\mathrm{M}$, et al. Recessive mutations in PCBD1 cause a new type of early-onset diabetes. Diabetes. 2014;63:3557-64.

20. Whibley A, Urquhart J, Dore J, et al. Deletion of MAOA and MAOB in a male patient causes severe developmental delay, intermittent hypotonia and stereotypical hand movements. Eur J Hum Genet. 2010;18: 1095-9.

21. O'Leary RE, Shih JC, Hyland K, Kramer N, Asher YJT, Graham JM. De novo microdeletion of Xp11.3 exclusively encompassing the monoamine oxidase A and B genes in a male infant with episodic hypotonia: a genomics approach to personalized medicine. Eur J Med Genet. 2012;55:349-53.

22. Saito M, Yamagata T, Matsumoto A, et al. MAOA/B deletion syndrome in male siblings with severe developmental delay and sudden loss of muscle tonus. Brain Dev. 2014;36:64-69.

23. Corzo D, Gibson W, Johnson K, et al. Contiguous deletion of the X-linked adrenoleukodystrophy gene (ABCD1) and DXS1357E: a novel neonatal phenotype similar to peroxisomal biogenesis disorders. Am J Hum Genet. 2002;70:1520-31.
24. Parvari R, Brodyansky I, Elpeleg O, Moses S, Landau D, Hershkovitz E. A recessive contiguous gene deletion of chromosome 2 p16 associated with cystinuria and a mitochondrial disease. Am J Hum Genet 2001;69:869-75.

25. Jaeken J, Martens K, Francois I, et al. Deletion of PREPL, a gene encoding a putative serine oligopeptidase, in patients with hypotonia-cystinuria syndrome. Am J Hum Genet. 2006;78:38-51.

26. Blau N, Duran M, Gibson KM, Dionisi-Vici C, editors. Physician's guide to the diagnosis, treatment, and follow-up of inherited metabolic diseases. 1st ed. Heidelberg: Springer; 2014.

27. Saudubray J-M, Baumgartner MR, Walter J, editors. Inborn metabolic diseases-diagnosis and treatment. 6th ed. Berlin: Springer; 2016.

28. Sarafoglou K, Hoffmann GF, Roth KS. Pediatric endocrinology and inborn errors of metabolism. 2nd ed. New York: McGraw-Hill; 2017.

29. Sollie $A$, Sijmons $R H$, Lindhout $D$, et al. A new coding system for metabolic disorders demonstrates gaps in the international disease classifications ICD-10 and SNOMED-CT, which can be barriers to genotype-phenotype data sharing. Hum Mutat. 2013;34:967-73.

30. Amberger IS, Bocchini CA, Schiettecatte F, Scott AF, Hamosh A. OMIM.org: Online Mendelian Inheritance in Man (OMIM $\left.{ }^{\oplus}\right)$, an online catalog of human genes and genetic disorders. Nucleic Acids Res. 2015;43:D789-798.

31. Saudubray J-M, Charpentier C. Clinical phenotypes: diagnosis/ algorithms. In: Beaudet AL, Vogelstein B, Kinzler KW, et al., editors. The online metabolic and molecular bases of inherited disease. New York: McGraw-Hill; 2014. ommbid.mhmedical.com/content.aspx? aid=1102922807. Accessed 11 January 2018.

32. Zschocke J. SSIEM classification of inborn errors of metabolism. In: Blau N, Duran M, Gibson KM, Dionisi Vici C, editors. Physician's guide to the diagnosis, treatment, and follow-up of inherited metabolic diseases. Berlin, Heidelberg: Springer; 2014. p. 817-30. 